Revista de Matemática: Teoría y Aplicaciones 2003 10(1-2) : 92-105

CIMPA - UCR - CCSS ISSN: 1409-2433

\title{
SOBRE EL PROBLEMA INVERSO DE DIFUSIÓN
}

\author{
J. R. Mercado E.* \\ Á. A. Aldama R. ${ }^{* *}$ \\ F. BRAmBILA P. ${ }^{* * *}$
}

Recibido: 21 May 2003

\begin{abstract}
Resumen
Se describe físicamente la infiltracción para modelarla como un proceso estocástico de difusión. Se enuncia el teorema M-B 1, cuyo objeto principal es el problema inverso de difusión. Se demuestra dicho teorema, en el contexto particular de la inyectividad de la solución y se aplica para resolver el problema inverso de difusión en presencia del grupo de Boltzmann. Se resuelve el problema inverso del exponente de similaridad por los métodos del análisis de grupo. Se aplica a la dispersión de una gota en un medio poroso tridimensional, resultado a su vez aplicable en el caso del riego por goteo.
\end{abstract}

Palabras clave: Problemas inversos, análisis de grupo de ecuaciones diferenciales, similaridad, fractales, difusión, medios porosos.

\begin{abstract}
Infiltration is physically described in order to model it as a diffusion stochastic process. Theorem M-B 1 is enunciated; whose main objective is the inverse diffusion problem. The theorem is demonstrated in the specific context of solution injectability, and it is applied to solve the inverse diffusion problem in the presence of Boltzmann's group. The inverse problem of the similarity exponent is solved following group analysis methods. The dispersion of a water drop in a three-dimensional porous medium is applied; a result which in turn is applicable to drop irrigation.
\end{abstract}

Keywords: inverse problems, group analysis of differential equations, similarity, fractals, diffusion, porous medium.

Mathematics Subject Classification: 35R30,28A80,76R99

\footnotetext{
*Instituto Mexicano de Tecnología del Agua, (IMTA), Coordinación de Tecnología Hidrológica, Paseo Cuauhnáhuac No. 8532, Progreso, Jiutepec, Morelos, México. Tel/Fax: (52)-(777)-319-35-44, Tel com: 3293600,ext 605 ó 532. E-Mail: rmercado@tlaloc.imta.mx

${ }^{* *}$ Misma dirección que R. Mercado. E-Mail: aaldama@tlaloc.imta.mx

*** Facultad de Ciencias, Universidad Nacional Autónoma de México (UNAM), 04510 México, D.F.. tel (5)-6224858. E-Mail: fbp@hp.fciencias.unam.mx
} 


\section{Introducción}

Se sabe que los procesos estocásticos pueden verse como la abstracción matemática de los procesos empíricos cuyas variables siguen un comportamiento aleatorio, [4].

Dentro de los procesos estocásticos, son de especial importancia aquellos de carácter unigeneracional, conocidos como procesos de Márkov, para los que se cumple la ecuación de Chapman-Kolmogórov y conducen a la ecuación de Fokker-Planck. Esta ecuación tiene tres fenómenos componentes: el difusivo, el advectivo y el reactivo. Desde un punto de vista más general, la ecuación de difusión clásica es una ecuación de Fokker-Planck asociada con un movimiento browniano standard, para el que la velocidad advectiva es cero y el coeficiente de difusión es constante normalizado al valor 1/2, [4], [10].

Por otra parte, en el estudio del problema inverso de dispersión en física, el potencial aparece como un coeficiente en la ecuación diferencial, por lo que deviene genérico, el llamar problema inverso a la obtención de los coeficientes de las diversas ecuaciones diferenciales, $[3]$.

Cuando el fenómeno difusivo es el dominante o incluso con la contribución del fenómeno advectivo constante o promediado, se requiere abordar inicialmente el problema inverso de difusión, para modelar dicho coeficiente y luego poder estudiar el problema directo de difusión. Este problema inverso de difusión es el objeto principal del teorema M-B 1, que se enuncia, se demuestra en un contexto particular y se aplica en el presente trabajo.

El teorema da la solución para el problema inverso de difusión en términos fractales.

En resumen, muchos fenómenos empíricos siguen la ecuación de Chapman-Kolmogórov y ésta conduce al estudio del coeficiente de difusión que se aborda con el teorema M-B 1.

En un trabajo publicado, [7], se aplicó el teorema citado y los métodos del análisis de grupo de las ecuaciones diferenciales, [8], al estudio de problemas inversos que surgen de la ecuación de Fokker-Planck. Allí se excluyó el caso del grupo de Boltzmann, tema denominado por algunos autores como difusión normal, pero en el presente trabajo, se aborda el análisis de esa situación para el problema inverso de difusión.

Posteriormente, se ilustra su importancia en los fenómenos de filtración del agua en medios porosos, como uno de los muchos ámbitos de aplicación y se resuelve el problema directo por métodos fractales.

Con métodos de la teoría de grupos de Lie, aplicados al problema inverso del exponente de similaridad, también se encuentran soluciones al problema directo de difusión, aplicables a muchos campos de la modelación matemática, como son: el drenaje horizontal, el drenaje vertical, la dispersión de una gota en el suelo y la dispersión de insectos, [6], [5].

\section{Modelación estocástica}

Imaginamos, de forma Lagrangiana, una pequeña gotita de agua del tamaño de los poros medios (tal vez del orden de las cien micras), que en el instante $t$ ocupa la posición $\xi_{t}$ y cambia de lugar en el futuro inmediato, para ocupar en el instante $t+\Delta t$ la posición $\xi_{t+\Delta t}$. Este cambio de posición o desplazamiento obedece a dos factores, uno de ellos es el movimiento debido al campo externo de la gravedad, que produce un arrastre global, el cual no tiene carácter aleatorio, sino que es de tipo determinista. Si el campo externo 
está representado por la velocidad de arrastre $a$ entonces este desplazamiento se representa por $a \Delta t$, y es el movimiento ordenado de la gotita de agua, el cual tiene dirección, que es la misma dirección de $a$. El otro desplazamiento está ligado a la tensión superficial de la capilaridad, que ejercen sobre la gotita los poros adyacentes a ella, y producen sobre ésta un desplazamiento de tipo aleatorío. Éste puede cuantificarse como el número de los desplazamientos relativos o pasos en una dirección por el valor del desplazamiento relativo o paso, menos el número de desplazamientos relativos en la dirección opuesta por el valor del mismo desplazamiento relativo. Por ejemplo seis pasos hacia adelante y cuatro pasos hacia atrás, producen dos pasos hacia adelante. El valor del paso se puede cuantificar por la distancia recorrida por unidad de la raíz cuadrada del intervalo de tiempo, multiplicada por la misma raíz cuadrada del intervalo de tiempo; entonces se tiene la raíz cuadrada del coeficiente de difusión, por el número de pasos, por la raíz cuadrada del intervalo de tiempo, en la dirección positiva $\left(\sigma B_{t+\Delta t}\right)$; menos lo análogo, pero en la dirección negativa $\left(\sigma B_{t}\right)$, el balance es entonces $\left(\sigma B_{t+\Delta t}-\sigma B_{t}\right)$. Éste es el movimiento desordenado, que no tiene o no depende de la dirección y tiene carácter aleatorio o caótico. De tal manera que

$$
\xi_{t+\Delta t}-\xi_{t} \approx a\left(t, \xi_{t}\right) d t+\sigma\left(t, \xi_{t}\right)\left(B_{t+\Delta t}-B_{t}\right)
$$

el cual se escribe

$$
d \xi_{t}=a\left(t, \xi_{t}\right) d t+\sigma\left(t, \xi_{t}\right) d B_{t} .
$$

De manera más formal, un proceso de difusión es un proceso estocástico:

$$
[0, \infty) \times \Omega:(s, \omega) \stackrel{\xi}{\mapsto} \xi(s, \omega)=\xi_{s}(\omega) \in \mathbb{R}^{n}
$$

que satisface una ecuación diferencial de la forma:

$$
d \xi_{s}=a\left(\xi_{s}\right) d s+\sigma\left(\xi_{s}\right) d B_{s}, s \geq t ; \quad \xi_{t}=x,
$$

donde $B_{s}$ es un movimiento Browniano $m$-dimensional y los coeficientes:

$$
a: \mathbb{R}^{n} \longrightarrow \mathbb{R}^{n}, \sigma: \mathbb{R}^{n} \longrightarrow \mathbb{R}^{n \times m},
$$

satisfacen las condiciones:

$$
\begin{array}{cccc}
|a(x)|+|\sigma(x)| & \leq & C_{1}(1+|x|) ; & |\sigma|^{2}=\Sigma\left|\sigma_{i j}\right|^{2} \\
|a(x)-a(y)|+|\sigma(x)-\sigma(y)| & \leq & C_{2}|x-y| ; & x, y \in \mathbb{R}^{n} .
\end{array}
$$

Una difusión es un proceso de Márkov y como tal corresponde a una modelación estocástica unigeneracional, que se basa principalmente en la ecuación de Chapman-Kolmogórov, según la cual el promedio es independiente de la gama de los valores intermedios de la variable aleatoria:

$$
E\left[\mu\left[\xi_{t} \in B \mid \xi_{\tau}\right] \mid \xi_{t_{0}}\right]=\mu\left[\xi_{t} \in B \mid \xi_{t_{0}}\right], t_{0}<\tau<t,
$$

donde $B$ es cualquier conjunto boreliano. Expresada en su forma integral y con respecto a la probabilidad de transición, las cuales se definen por

$$
F_{t}\left(B \mid \xi_{t_{0}}(\omega)=x_{0}\right)=\mu\left[\xi_{t}(\omega) \in B \mid \xi_{t_{0}}(\omega)=x_{0}\right]=p\left(t_{0}, x_{0}, t, B\right),
$$

es

$$
u\left(t_{0}, x_{0}, t, B\right)=\int u\left(t_{0}, x_{0}, \tau, x_{\tau}\right) \cdot u\left(\tau, x_{\tau}, t, B\right), t_{0}<\tau<t
$$




\subsection{El generador del semigrupo}

El semigrupo de operadores se define por su acción sobre las mediciones o funciones medibles y acotadas con dominio en el espacio fásico y rangos en los reales

$$
T_{t+\tau} f\left(x_{0}\right)=\int_{X} u\left(t+\tau, x_{0}, d x\right) f(x),
$$

esto conlleva a definir los coeficientes de memoria del proceso como los promedios:

$$
a=\lim _{\tau \rightarrow 0} \int_{X} u(t+\tau, \bar{x}, d x) \frac{(x-\bar{x})}{\tau}, \quad D=\lim _{\tau \rightarrow 0} \int_{X} u(t+\tau, \bar{x}, d x) \frac{1}{2} \frac{(x-\bar{x})^{2}}{\tau},
$$

y se obtiene el generador del semigrupo asociado a la difusión $\xi_{t}$, el cual es un operador diferencial parcial de segundo orden $L$, llamado el generador infinitesimal de la difusión $\xi_{t}$

$$
L=-\partial_{x}(a u)+\partial_{x x}(D u)+f .
$$

Una forma alternativa es la ecuación con término de reacción o fuente, ya que es posible definir un proceso estocástico asociado que resulta también un proceso de Márkov fuerte, y cuyo generador es, [10],

$$
\begin{gathered}
\tilde{L}=L-f . \\
L=-\partial_{x}(a u)+\partial_{x x}(D u)+f .
\end{gathered}
$$

Si se considera a $u$ como la densidad de probabilidad de una variable aleatoria y a $J$, la corriente de probabilidad de la misma variable,

$$
J=\left(a-\frac{\partial}{\partial x} D\right), \mathrm{y} \frac{\partial}{\partial t} u=-\frac{\partial}{\partial x} J u+f,
$$

entonces la ecuación de Fokker-Planck es:

$$
\frac{\partial}{\partial t} u=L u
$$

La ecuación de Fokker-Planck lineal, se transforma en la ecuación de difusión, advención y reacción, y adquiere la siguiente forma,

con $-a+D_{x} \mapsto-a$,

$$
\frac{\partial}{\partial t} u=\frac{\partial}{\partial x}\left(D(x) \frac{\partial}{\partial x} u\right)-a(x) \frac{\partial}{\partial x} u-a_{x} u ; \quad u \in \mathcal{C}_{0}^{2}(\mathbb{R}),
$$

y la correspondiente ecuación de Fokker-Planck no lineal es

$$
\frac{\partial}{\partial t} u=\frac{\partial}{\partial x}\left(D(u) \frac{\partial u}{\partial x}\right)-a(u) \frac{\partial}{\partial x} u+f(u) ; \quad u \in \mathcal{C}_{0}^{2}(\mathbb{R}) .
$$

Esta ecuación es de tipo parabólico y contiene tres ingredientes principales, los cuales pueden llamarse: el difusivo, el advectivo y el reactivo; y por lo general se habla de advección con $-a, \mathrm{y}$ de convección con $+a$. 
Por tanto, una plétora de procesos empíricos pueden modelarse con fundamento en procesos estocásticos que presentan por ejemplo, la ausencia del efecto posterior, no tienen memoria o son unigeneracionales; éstos conducen a una ecuación de Chapman-Kolmogórov, de ésta se llega a la ecuación de Fokker-Planck y por tanto, a considerar los problemas inversos de difusión, advección y reacción, [7].

\section{Método fractal}

El problema inverso de difusión se aborda desde el punto de vista del teorema MB1, [7], [9]:

Teorema 1 (M-B 1) El inverso del coeficiente de difusión se obtiene por un proceso de Cantor generalizado:

$$
B=\lim _{n \rightarrow \infty}(-1)^{n} \partial_{p_{n}}^{(n)}\left(\frac{1}{q_{n}}\right) \cdot(n+1) \partial_{p_{n}}^{(-n)}\left(p_{n}\right),
$$

en donde $\partial_{p_{n}}^{(n)}$ es la enésima derivación sucesiva, $\partial_{p}^{(-n)}=\int^{(n)}$ la enésima integración sucesiva, $q_{n}$ viene determinada por la función de los datos en el problema inverso, se asocia al hipervolumen en el proceso de Cantor y $\frac{1}{q_{n}}$ es la probabilidad de paso del proceso de percolación. Finalmente, $p_{n}$ es el número de rasgos o elementos $\frac{1}{q_{n}}$ en el proceso de fractalización de Cantor y se le asocia a la masa, $(D=1 / B$ tiene unidades físicas de $\left.m t^{2} / \mathrm{seg}\right)$.

Este teorema se demuestra en el contexto particular de la inyectividad de la variable independiente o humedad $u(x, t)$, como función de $x$, y se denota la inversa por la izquierda como $x(u, t)$.

Se considera un grupo local de transformaciones $G$ dado a través de su generador infinitesimal, [11],

$$
\mathbf{v}=\xi \partial_{x}+\tau \partial_{t}+\psi \partial_{u}
$$

bajo el cual la ecuación diferencial de difusión quede invariante; éste puede ser, por ejemplo, un grupo de escalas. Se define el grupo por los cambios

$$
x \longmapsto \beta x, \quad t \longmapsto \gamma t, \quad u \longmapsto \delta u,
$$

y la invarianza de la ecuación se garantiza si

$$
\gamma=\beta^{2}, \quad \delta=1,
$$

y el grupo se describe por

$$
\begin{aligned}
\tilde{x} & =\beta x=e^{\varepsilon} x=x+\varepsilon x+O\left(\varepsilon^{2}\right) \\
\tilde{t} & =\gamma t=e^{2 \varepsilon} t=t+\varepsilon(2 t)+O\left(\varepsilon^{2}\right) \\
u & =\delta u=u+\varepsilon 0,
\end{aligned}
$$


por tanto su generador es

$$
\mathbf{v}=x \partial_{x}+2 t \partial_{t}+0 \partial_{u}
$$

y de las ecuaciones características, se obtiene la variable de similaridad,

$$
\phi=\frac{x}{\sqrt{t}}
$$

en donde a $\phi(u)$ se le conoce como variable de similaridad de Boltzmann. Por tanto, se tiene a $x$ en función de la variable $\phi(u)$

$$
x(u, t)=\phi(u) \sqrt{t} .
$$

Se considera a la función humedad $u$ definida implícitamente a través de la función $x(u, t), \mathrm{y}$ con el teorema de la función implícita se halla la relación:

$$
\left(\frac{\partial u}{\partial t}\right)_{x}=-\frac{\left(\frac{\partial x}{\partial t}\right)_{u}}{\left(\frac{\partial x}{\partial u}\right)_{t}}
$$

luego la ecuación de continuidad se transforma en una ecuación de difusión:

$$
\frac{\partial x}{\partial t}+\frac{\partial}{\partial u}\left(\frac{D(u)}{\partial x / \partial u}\right)=0, \quad \frac{\phi}{2 \sqrt{t}}+\frac{\partial}{\partial u}\left(\frac{D(u)}{\phi^{\prime}(u) \sqrt{t}}\right)=0,
$$

de donde puede obtenerse el coeficiente de difusividad, [13]:

$$
D(u)=-\frac{1}{2} \phi^{\prime}(u) \int_{u_{0}}^{u} \phi d \bar{u} .
$$

Además, como $\phi$ es positiva (no-negativa), la integral también es positiva, luego la derivada debe ser negativa, para obtener una difusividad positiva, entonces $\phi$ debe ser decreciente. La integral aporta el factor más regular y la derivada el factor menos regular o "irregular".

Se discretiza la variable $\phi$ y resulta la sucesión

$$
D_{n}(u)=-\frac{1}{2} \phi_{n}^{\prime}(u) \int_{u_{0}}^{u} \phi_{n} d \bar{u},
$$

si se denota con $I_{n}$ a la integral, el resultado encontrado para este coeficiente de difusión, puede presentarse como:

$$
\sqrt{D_{n}} \sqrt{t_{n}} \cdot \frac{\sqrt{D_{n}}}{\sqrt{t_{n}}}=\left(-\frac{1}{2} \phi_{n}^{\prime}\right) \cdot I_{n} .
$$

Por otra parte, en la descripción del proceso de infiltración, la parte del desplazamiento que tiene carácter aleatorio, lo representamos por el producto entre el valor del paso $-\sqrt{D} \sqrt{t}-$, y el número de pasos $-\frac{B_{t}}{\sqrt{t}^{-}}$, luego, por:

$$
\sqrt{D_{n}} \sqrt{t_{n}} \cdot \frac{B_{t_{n}}}{\sqrt{t_{n}}}=\sqrt{D_{n}} \cdot B_{t_{n}}
$$


Y, por una tercera parte, la fractalización la podemos describir como un proceso de fractura y conteo de las partes, la fractura la realiza la diferenciación, y el conteo lo hace la integración; de suerte que en cada etapa del proceso las medidas de las partes son $\frac{(-1)^{n}}{n !} \partial_{p_{n}}^{(n)}\left(\frac{1}{q_{n}}\right)$ y el número de rasgos o partes son: $(n+1) ! \partial_{p_{n}}^{(-n)}\left(p_{n}\right)$. Al tomar en cuenta las unidades físicas de $B$ como seg $/ \mathrm{mts}^{2}$, la medida del conjunto, entonces es

$$
\frac{1}{\frac{(-1)^{n}}{n !} \partial_{p_{n}}^{(n)}\left(\frac{1}{q_{n}}\right)} \cdot \frac{1}{(n+1) ! \partial_{p_{n}}^{(-n)}\left(p_{n}\right)}
$$

en donde a $q$ lo llamamos la base de la resolución y a $p$ los rasgos.

A la porción aleatoria de la descripción de la infiltracción se le asocia dos representaciones de una misma medida, que se expresan por el producto de una variable intensiva o densidad, y por una variable extensiva, o bien, es una medida absolutamente continua, expresada como el producto entre la densidad y la medida extensiva, siendo la densidad única. Por tanto se tiene la correspondencia

$$
\sqrt{D_{n}} \cdot B_{t_{n}} \mapsto\left(-\frac{1}{2} \phi_{n}^{\prime}\right) \cdot I_{n}=\frac{1}{\frac{(-1)^{n}}{n !} \partial_{p_{n}}^{(n)}\left(\frac{1}{q_{n}}\right)} \cdot \frac{1}{(n+1) ! \partial_{p_{n}}^{(-n)}\left(p_{n}\right)}
$$

de donde se obtiene por la unicidad de la densidad

$$
-\frac{1}{2} \phi_{n}^{\prime}=\frac{1}{\frac{(-1)^{n}}{n !} \partial_{p_{n}}^{(n)}\left(\frac{1}{q_{n}}\right)}
$$

y el factor irregular resulta asociado a la fase de la resolución de la fractalización, la cual es además positiva. Como el factor irregular se comporta en forma creciente, entonces esta fase es decreciente en el proceso de fractalización, y recíprocamente. En tanto, el factor regular se relaciona entonces, con la fase de los rasgos de la fractalización, la cual también es positiva

$$
\frac{1}{(n+1) ! \partial_{p_{n}}^{(-n)}\left(p_{n}\right)}=I_{n}=\int_{u_{0}}^{u} \phi_{n}(\bar{u}) d \bar{u},
$$

el factor regular se comporta en forma decreciente, correspondiendo al comportamiento creciente de la fase de los rasgos.

Por tanto el coeficiente de difusión es el límite de la sucesión $D_{n}$, y ésta es el producto de la sucesión de la fase de la resolución por la sucesión de la fase de los rasgos del proceso de fractalización

$$
\begin{gathered}
D=\lim _{n} D_{n} \\
D_{n}=\frac{1}{\frac{(-1)^{n}}{n !} \partial_{p_{n}}^{(n)}\left(\frac{1}{q_{n}}\right)} \cdot \frac{1}{(n+1) ! \partial_{p_{n}}^{(-n)}\left(p_{n}\right)} .
\end{gathered}
$$

Se observa además, que las sucesiones de los infinitesimales del generador del grupo de transformaciones, determinan las otras dos sucesiones, la de la resolución y la de los rasgos, 
lo cual se hace explícito a través de las relaciones

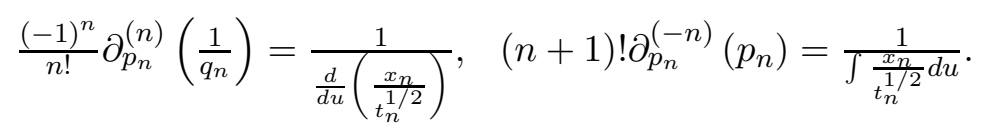

\subsection{Aplicación}

Como aplicación se puede encontrar la difusividad y resolver el problema directo de la ecuación de difusión, sujeta a las condiciones límites, inicial:

$$
u(x, 0)=u_{0}=\text { cte } \Leftrightarrow \phi\left(u_{0}\right) \rightarrow \infty
$$

y de frontera:

$$
\begin{aligned}
u(0, t) & =u_{s}=\text { cte } \Leftrightarrow \phi\left(u_{s}\right)=0, \mathrm{y} \\
u(\infty, t) & =u_{0}=\text { cte } .
\end{aligned}
$$

Según el procedimiento de Phillips, [13], para el problema inverso, se debe escoger una variable de similaridad apropiada; pero de acuerdo con nuestro procedimiento se debe escoger una base de resolución en concordancia con el modelo físico y construir el factor irregular, que permita hallar la sucesión de la variable de similaridad, y finalmente en el límite del proceso de fractalización, obtener la variable de similaridad. Sea

$$
q=1+p
$$

entonces la fase de la resolución decrece como una potencia

$$
\frac{(-1)^{n}}{n !} \partial_{p}^{(n)}\left(\frac{1}{q}\right)=\frac{1}{q^{n+1}}=\frac{1}{(1+p)^{n+1}}
$$

se discretiza con $p_{n}=b \frac{u}{n}$, y se modela el fenómeno como la competencia entre el flujo y la retención, considerándose que para una etapa avanzada de la fractalización predomina la retención. Así que para la base de la resolución cuando $n$ es suficientemente grande queda el término ligado a la retención. Por tanto, el factor irregular crece como una potencia

$$
\begin{aligned}
-\frac{1}{2} \phi_{n}^{\prime}(u) & =\left(1+b \frac{u}{n}\right)^{n+1} \\
\phi_{n}(u) & =-\frac{2}{b} \frac{n}{n+2}\left(1+b \frac{u}{n}\right)^{n+2}+c t e_{n},
\end{aligned}
$$

y de acuerdo a la condición límite $\phi\left(u_{s}\right)=0$

$$
c t e_{n}=\frac{2}{b} \frac{n}{n+2}\left(1+b \frac{u_{s}}{n}\right)^{n+2}
$$

luego

$$
\phi_{n}(u)=\frac{2}{b} \frac{n}{n+2}\left(\left(1+b \frac{u_{s}}{n}\right)^{n+2}-\left(1+b \frac{u}{n}\right)^{n+2}\right)
$$


y en el límite, con $b=c t e>0$, resulta:

$$
\phi(u)=\frac{2}{b}\left(e^{b u_{s}}-e^{b u}\right) .
$$

Ahora puede calcularse el coeficiente de difusividad, [2],

$$
\begin{gathered}
\phi^{\prime}(u)=-2 e^{b u} \\
\int_{u_{0}}^{u} \phi(\bar{u}) d \bar{u}=-\frac{2}{b^{2}}\left(e^{b u}-e^{b u_{0}}\right)+\frac{2}{b} e^{b u_{s}}\left(u-u_{0}\right)
\end{gathered}
$$

luego

$$
D(u)=e^{b u}\left(-\frac{2}{b^{2}}\left(e^{b u}-e^{b u_{0}}\right)+\frac{2}{b} e^{b u_{s}}\left(u-u_{0}\right)\right)
$$

y se ilustra su gráfica en la figura 1.

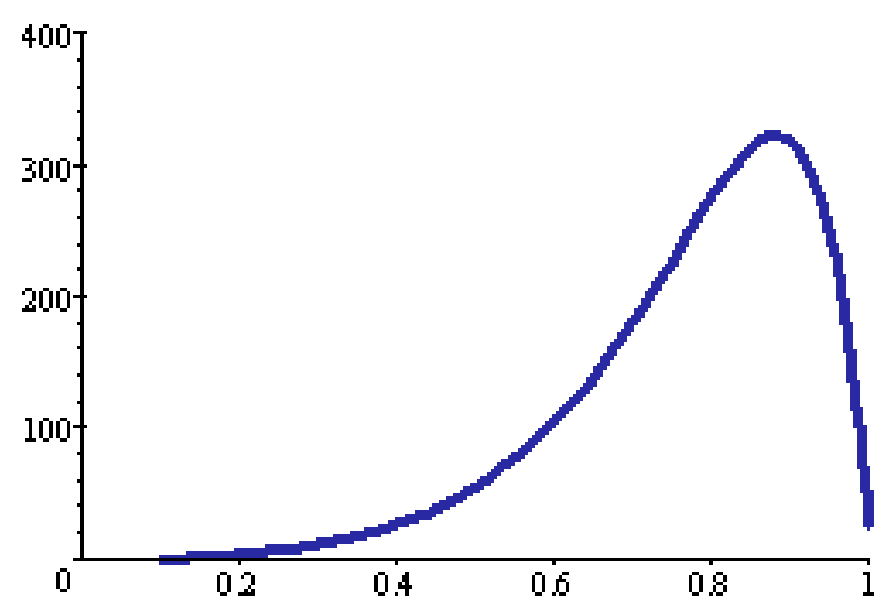

Figura 1: Difusividad.

Las soluciones autosimilares se encuentran por

$$
\frac{x}{\sqrt{t}}=\phi=\frac{2}{b}\left(e^{b u_{s}}-e^{b u}\right)
$$

entonces

$$
u(x, t)=\frac{1}{b} \ln \left(-\frac{b x}{2 \sqrt{t}}+e^{b u_{s}}\right),
$$

que tiene la forma similar a un flujo pistón que avanza progresivamente, como se ilustra en su gráfica (2).

Alternativamente, del factor regular se obtiene, con $a_{n}=\frac{2}{b} \frac{n}{n+2}$,

$$
\int_{u_{0}}^{u} \phi_{n}(\bar{u}) d \bar{u}=\left.a_{n}\left\{\left(1+b \frac{u_{s}}{n}\right)^{n+2} u-\frac{1}{n+3} \frac{n}{b}\left(1+b \frac{u}{n}\right)^{n+3}\right\}\right|_{u_{0}} ^{u}
$$




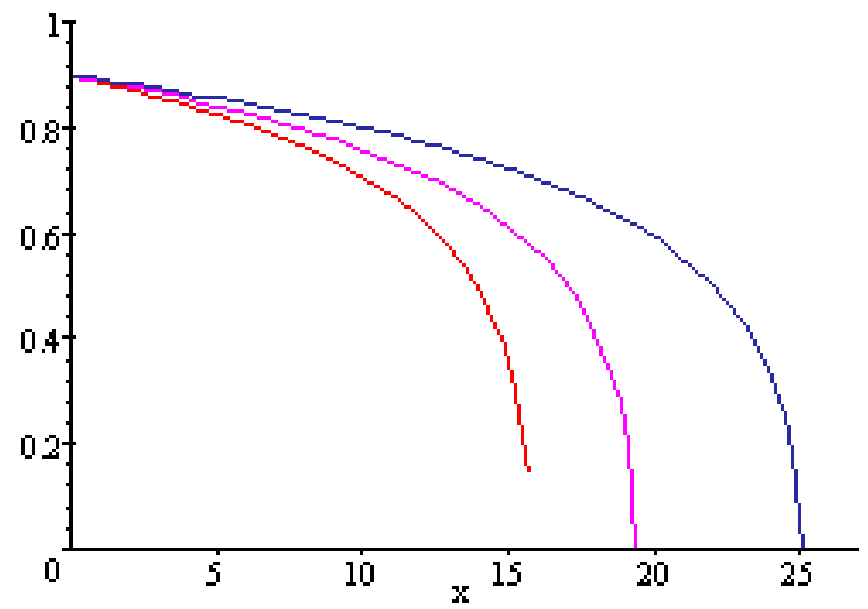

Figura 2: Soluciones autosimilares.

y al tomar el límite, y luego derivar, se puede también obtener la variable de similaridad $\phi$ dada por (26).

\section{El exponente de similaridad}

Cuando la ecuación de Fokker-Planck no lineal se somete a un análisis de grupo se produce la ecuación

$$
\left(\frac{D}{D^{\prime}}\right)_{u u}\left(\tau_{t}-2 \xi_{x}\right)=0
$$

ésta representa una disyuntiva, porque si $\tau_{t}-2 \xi_{x} \neq 0$, se puede determinar el coeficiente de difusión $D$, tal como se afirma en el teorema MB1, a partir de la base de la resolución construida en términos de los datos en una frontera especificada. Pero si el grupo presente es tal que $\tau_{t}-2 \xi_{x}=0$, entonces con el análisis de grupo no es posible determinar el coeficiente de difusión, aunque sí lo es por medio del teorema MB1, (27), [7]. Por su importancia en los fenómenos difusivos, a éste grupo lo hemos denominado el grupo de Boltzmann.

Cuando este grupo no está presente, y despúes de estudiar el problema inverso para el coeficiente de difusión, se obtiene como una posible solución la potencia del polinomio lineal, y luego de realizar una traslación en la variable dependiente, se llega a la ecuación de Boussinesq del medio poroso:

$$
\begin{aligned}
\Delta & =\frac{1}{x^{n-1}} \partial_{x}\left(x^{n-1} u^{m} \partial_{x} u\right)-\partial_{t} u \\
& =\frac{(n-1)}{x} u^{m} u_{x}+m u^{m-1} u_{x}^{2}+u^{m} u_{x x}-u_{t} ;
\end{aligned}
$$

para distintos valores de la potencia se obtienen casos importantes, como son: para $m=0$, la ecuación del calor de Fourier; para $m=1$, la ecuación para la filtración, que describe el abatimiento del manto freático; para $m=-2$, la difusividad de Fujita, quien tiene el 
mérito de ser el primero en resolver analíticamente la ecuación de difusión con coeficiente dependiente de la variable independiente, o en forma no-lineal.

Se realiza un análsis de grupo de esta ecuación, [1], [11]. El grupo se representa por su generador infinitesimal $\mathbf{v}$,

$$
\mathbf{v}=\xi \partial_{x}+\tau \partial_{t}+\phi \partial_{u}
$$

que induce una acción ampliada descrita por la sección campo vectorial 2-jet $j^{(2)} \mathbf{v}(\cdot)$

$$
j^{(2)} \mathbf{v}=\mathbf{v}+\phi^{x} \partial_{u_{x}}+\phi^{t} \partial_{u_{t}}+\phi^{x x} \partial_{u_{x x}}+\phi^{x t} \partial_{u_{x t}}+\phi^{t t} \partial_{u_{t t}} .
$$

Para las distintas dimensiones se encuentra que el infinitesimal asociado al tiempo siempre es

$$
\tau=2 \delta t+\sigma
$$

mientras que el grupo se determina por cuatro parámetros, dentro del cual pueden observarse los subgrupos de la traslación espacial y temporal, y los cambios de escala.

Con la condición de que las características pasen por el origen $x=0, t=0$, se tiene que el generador para las distintas dimensiones, asume la forma

$$
\begin{aligned}
\mathbf{v} & =(\beta+\delta) x \partial_{x}+2 \delta t \partial_{t}+\frac{2 \beta u}{m} \partial_{u} \\
& =\beta\left(x \partial_{x}+\frac{2}{m} u \partial_{u}\right)+\delta\left(x \partial_{x}+2 t \partial_{t}\right)
\end{aligned}
$$

salvo para los exponentes $m=-\frac{4}{3}$, en la dimensión $n=1 ; m=-1$, en la dimensión $n=2 ; \frac{1}{m}=\frac{n-4}{-2(n-3)}$ en la dimensión $n>3$.

\subsection{Variables de similaridad}

Con el análisis de las ecuaciones características se busca determinar las variables de similaridad. Las ecuaciones de dichas curvas son

$$
\frac{d x}{(\beta+\delta) x}=\frac{d t}{2 \delta t}=\frac{d u}{\frac{2 \beta u}{m}},
$$

por tanto resulta

$$
x=c t e_{2} \cdot t^{\frac{1}{2}+\frac{\beta}{2 \delta}}, \quad u=c t e_{1} \cdot t^{\frac{\beta}{\delta m}} .
$$

Cuando $t \rightarrow \infty$ debe tenerse que $u \rightarrow 0$, luego $\frac{\beta}{\delta}$ es menor que cero, y si

$$
\frac{\beta}{\delta}=-\alpha, \alpha>0
$$

se tiene entonces para las curvas características

$$
u=c t e_{1} \cdot t^{-\frac{\alpha}{m}}, \quad x=c t e_{2} \cdot t^{\frac{1}{2}-\frac{\alpha}{2}} .
$$

Se define las variables de similaridad por

$$
v=u \cdot t^{\frac{\alpha}{m}}, \quad y=x \cdot t^{-\frac{1}{2}+\frac{\alpha}{2}}
$$


y entonces la solución se representa de la forma

$$
u=t^{-\frac{\alpha}{m}} v\left(x \cdot t^{-\frac{1}{2}+\frac{\alpha}{2}}\right)
$$

llamada por algunos autores, la solución autosimilar de segunda clase.

Al sustituir en la ecuación diferencial las derivadas requeridas y aplicar la condición de invarianza, se obtiene la llamada ecuación auxiliar

$$
0=\frac{\alpha}{m} v+\left(a y+\frac{(n-1)}{y} v^{m}\right) v^{\prime}+v^{m} v^{\prime \prime}+m v^{m-1}\left(v^{\prime}\right)^{2}
$$

la cual resulta independiente de la variable $t$, y en donde se ha denotado $a=-\frac{1}{2}+\frac{\alpha}{2}$.

Como en el caso del estudio de la ecuación de Boussinesq para la filtración, para $m=1$ y dimensión $n=1$, se obtiene la ecuación que llamamos especial

$$
0=\alpha v+v^{\prime}\left(\frac{1}{2}(-1+\alpha)\right) y+v v^{\prime \prime}+\left(v^{\prime}\right)^{2} .
$$

Se hace uso nuevamente de la teoría del análisis de grupo de las ecuaciones diferenciales, pero en este caso, para ecuaciones diferenciales ordinarias, y se obtiene los infinitesimales

$$
\xi=y, \quad \phi=2 v,
$$

en donde se observa que corresponden a un cambio de escala, con el generador representado por

$$
\mathbf{v}=y \partial_{y}+2 v \partial_{v}
$$

Se introducen las coordenadas canónicas:

$$
\left(\begin{array}{l}
x \\
u
\end{array}\right) \longmapsto\left(\begin{array}{l}
y \\
w
\end{array}\right)=\left(\begin{array}{l}
\frac{u}{x^{2}} \\
\ln x
\end{array}\right)
$$

para transformar la ecuación diferencial especial, con $w_{y}=z$, en

$$
z^{\prime}=(6 y+1) z^{3}+\left(7-\frac{a}{y}\right) z^{2}+\frac{1}{y} z
$$

la cual es la ecuación reducida al primer orden. En la singularidad $w_{y}=\infty$ o $y_{w}=0$, se obtiene que $y$ como función de $w$ es constante: $y=-\frac{1}{6}$, y con $u=y x^{2}$, se tiene $u=-\frac{1}{6} x^{2}$, sin embargo, para representar magnitudes físicas como se requieren en las aplicaciones, se realiza una traslación positiva suficiente, y con $u=c\left(b^{2}-x^{2}\right)$ en la ecuación auxiliar especial, se obtiene

$$
c=\frac{1}{6}, \quad \alpha=\frac{1}{3},
$$

con lo que se tiene la solución buscada para el problema inverso del coeficiente $\alpha$, en el caso importante de la ecuación auxiliar especial. Además, la solución de similaridad resulta

$$
u=\frac{1}{6}\left(b^{2}-x^{2}\right)
$$


la cual tiene la forma de una familia de parábolas que se abren hacia abajo.

Para el caso general, se introduce el cambio de variable

$$
u^{m}=v
$$

y con

$$
v=c\left(b^{2}-x^{2}\right)
$$

resulta entonces

$$
c=\frac{1}{2\left(\frac{2}{m}+n\right)} ; \alpha=\frac{n}{2+m n} ; m \neq-\frac{2}{n}, 0,
$$

con lo que se obtiene la solución para el problema inverso del coeficiente $\alpha$ de la ecuación auxiliar del medio poroso.

\subsection{Aplicación}

Para el caso de dimensión $n=3$, se quiere representar la dispersión de una gota en un medio poroso.

En las variables originales, $u=t^{-\frac{\alpha}{m}} v\left(r t^{-\frac{1}{2}+\frac{\alpha}{2}}\right)$, se tiene

$$
\begin{aligned}
& u=t^{-\frac{3}{2+3 m}} v\left(r t^{-\frac{1}{2+3 m}}\right), y=r t^{-\frac{1}{2+3 m}} \\
& v=\left(c\left(b^{2}-y^{2}\right)\right)^{\frac{1}{m}}=\left(\frac{1}{2\left(\frac{2}{m}+3\right)}\left(b^{2}-\left(\frac{r}{\frac{1}{t^{2+3 m}}}\right)^{2}\right)\right)^{\frac{1}{m}},
\end{aligned}
$$

entonces las soluciones de similaridad son:

$$
u(r, t ; b)=0 \vee \frac{1}{\frac{3}{t^{2+3 m}}}\left(\frac{m}{2(2+3 m)}\left(b^{2}-\frac{r^{2}}{\frac{2}{t^{2+3 m}}}\right)\right)^{\frac{1}{m}} .
$$

Se puede lograr una presentación más simétrica, al sustituir la constante positiva $b^{2}$ por la también constante positiva $r_{0}^{2}$ a través de la relación

$$
b^{2}=\frac{r_{0}^{2}}{\frac{2}{t_{1}^{2+3 m}}} .
$$

Se asume la presencia de un coeficiente $D_{m}$ en el coeficiente de difusión, el cual se define en términos de la porosidad efectiva como $D_{m}=D_{s} \phi^{m}$, y en donde $D_{s}$ se entiende proporcional a la conductividad saturada. Se introduce la constante temporal $t_{1}$ y la constante espacial $r_{0}$

$$
t_{1}=\frac{m r_{0}^{2}}{2(2+3 m) D_{s}}, \quad r_{1}=r_{0}\left(\frac{t}{t_{1}}\right)^{\frac{1}{2+3 m}}
$$

y las soluciones autosimilares adquieren la forma, [5], [12]:

$$
u\left(r, t ; r_{0}\right)=\left\{\begin{array}{cc}
\frac{1}{\phi}\left(\frac{t_{1}}{t}\right)^{\frac{3}{2+3 m}}\left(1-\frac{r^{2}}{r_{1}^{2}}\right)^{\frac{1}{m}} & r \leq r_{1} \\
0 & r>r_{1}
\end{array} .\right.
$$


La constante $r_{0}$ puede determinarse a través de una condición de normalización. Se fija la cantidad de líquido que contiene la gota, como una muestra inicialmente concentrada, después el fenómeno de la difusión evoluciona y entonces la integral espacial de la concentración en cualquier otro momento posterior debe ser la cantidad original, suponiendo que no hay evaporación, luego:

$$
M(r, t)=\int_{0}^{r} u\left(r, t ; r_{0}\right) d V=\frac{2 \pi}{\phi}\left(\frac{t_{1}}{t}\right)^{\frac{3}{2+3 m}} \int_{0}^{r}\left(1-\frac{r^{2}}{r_{1}^{2}}\right)^{\frac{1}{m}} r^{2} d r
$$

y con el cambio de variable $y=\frac{r^{2}}{r_{1}^{2}}$, se obtiene la que fue denominada por Johann Carl Friedrich Gauss como la distribución beta. La evolución se da entonces por:

$$
M(r, t)=M_{0} \text { DistBeta }\left(\left(\frac{r}{r_{1}}\right)^{2} ; \frac{3}{2}, \frac{1}{m}+1\right)
$$

y la relación entre la masa inicial de agua y la distancia cubierta resulta ser:

$$
r_{0}=\left(\frac{\phi M_{0}}{\pi B\left(\frac{3}{2}, \frac{1}{m}+1\right)}\right)^{\frac{1}{3}}
$$

\section{Conclusiones}

- El método fractal del teorema MB1, es útil para resolver, tanto el problema inverso, como también el problema directo de difusión.

- El método fractal permite obtener explícitamente la variable de Boltzmann.

- El análisis de grupo de ecuaciones diferenciales da la posibilidad de resolver el problema inverso del exponente de similaridad en el modelo de la ecuación de Boussinesq.

- Creemos haber extendido el concepto de problema inverso, desde los coeficientes de una ecuación diferencial, hasta incluir los exponentes, como es el caso del exponente de similaridad.

\section{Referencias}

[1] Bluman, G.W.; Kumei, S. (1989) Symmetries and Differential Equations. SpringerVerlag, New York.

[2] Broadbridge, P.; Goard, J.M.; Lavrentiev Jr., M. (1997) "Degenerate nonlinear difussion with an initially sharp front", Studies in Applied Math. 99: 377-391.

[3] Colton, D.; Ewing, R.; Rundell, W. (Eds.) (1990) Inverse Problems in Partial Differential Equations. SIAM, Philadelphia. 
[4] Doob, J.L. (1960) Stochastic Processes. John Wiley \& Sons, New York.

[5] Galaktionov, V.A. (1994) "Blow-up for quasilinear heat equations with critical Fujita's exponents", Proceedings of the Royal Society of Edinburgh 124A: 517-525.

[6] Mercado, J.R.; Aldama, Á.A.; Brambila, F. (2002) "Sobre la ecuación de Boussinesq del medio poroso", Aportaciones Matemáticas, Serie Comunicaciones 30: 165-187.

[7] Mercado, J.R.; Brambila, F. (2001) "Problemas inversos en las ecuaciones de FokkerPlanck", Aportaciones Matemáticas, Serie Comunicaciones 29: 201-222.

[8] Mercado, J.R.; Hernández, G.; Ramos, L.J.; Ockendon, H.; Brambila, F. (1999) "Problemas directo e inverso para el abatimiento del manto freático", Aportaciones Matemáticas 23: 49-58.

[9] Mercado, J.R.; Namuche, R.; Fuentes, C.; Brambila, F. (1996) "Naturaleza fractal de la difusividad hidráulica", XVII Congreso Latinoamericano de Hidráulica, Guayaquil, Ecuador.

[10] Oksendal, B. (1989) Stochastic Differential Equations. Springer-Verlag, New York.

[11] Olver, P.J. (1993) Applications Lie Groups to Differential Equations. Springer-Verlag, Berlin.

[12] Pattle; R. E. (1959) "Difusion from an instantaneous point source with a concentration-dependent coefficient", Quart. Journ. Mech. and Appl. Math. 12(4): 407-409.

[13] Philip, J.R. (1960) "General method of exact solution of the concentration-dependent diffusion equation", Austral. J. Phys. 13: 1-12. 